\title{
Focal Pontine Lesions Provide Evidence That Intrinsic Functional Connectivity Reflects Polysynaptic Anatomical Pathways
}

\author{
Jie Lu, ${ }^{1 *}$ Hesheng Liu, ${ }^{3 *}$ Miao Zhang, ${ }^{1}$ Danhong Wang, ${ }^{3}$ Yanxiang Cao, ${ }^{1}$ Qingfeng Ma, ${ }^{2}$ Dongdong Rong, ${ }^{1}$ Xiaoyi Wang, \\ Randy L. Buckner, ${ }^{3,4,5,6}$ and Kuncheng $\mathrm{Li}^{1}$ \\ Departments of ${ }^{1}$ Radiology and ${ }^{2}$ Neurology, Xuanwu Hospital of Capital Medical University, Beijing 100053, China, ${ }^{3}$ Athinoula A. Martinos Center for \\ Biomedical Imaging, Department of Radiology and ${ }^{4}$ Department of Psychiatry, Massachusetts General Hospital, Charlestown, Massachusetts 02129, \\ ${ }^{5}$ Psychology and Center for Brain Science, Harvard University, Cambridge, Massachusetts 02138, and ${ }^{6}$ Howard Hughes Medical Institute, Cambridge, \\ Massachusetts 02138
}

Intrinsic functional connectivity detected by functional MRI (fMRI) provides a useful but indirect approach to study the organization of human brain systems. An unresolved question is whether functional connectivity measured by resting-state fMRI reflects anatomical connections. In this study, we used the well-characterized anatomy of cerebrocerebellar circuits to directly test whether intrinsic functional connectivity is associated with an anatomic pathway. Eleven first-episode stroke patients were scanned five times during a period of 6 months, and 11 healthy control subjects were scanned three times within 1 month. In patients with right pontine strokes, the functional connectivity between the right motor cortex and the left cerebellum was selectively reduced. This connectivity pattern was reversed in patients with left pontine strokes. Although factors beyond anatomical connectivity contribute to fMRI measures of functional correlation, these results provide direct evidence that functional connectivity depends on intact connections within a specific polysynaptic pathway.

\section{Introduction}

Intrinsic functional connectivity MRI (fcMRI) provides an indirect approach to measure the organization of brain systems (Biswal et al., 1995; for review, see Fox and Raichle, 2007; Cole et al., 2010; Van Dijk et al., 2010). The method takes advantage of spontaneous fluctuations in the blood oxygenation leveldependent fMRI signal that are coupled selectively between certain regions. Although the physiological basis of the slow intrinsic activity fluctuations that underlie fcMRI remain incompletely understood, the correlation patterns suggest that a major (but not exclusive) determinant of functional coupling is anatomic connectivity (Vincent et al., 2007; Greicius et al., 2009; Honey et al., 2009; Margulies et al., 2009). fcMRI has been widely used to understand the architecture of human brain systems during de-

Received May 11, 2011; revised Aug. 22, 2011; accepted Aug. 26, 2011.

Author contributions: J.L., H.L., R.L.B., and K.L. designed research; J.L., H.L., M.Z., Y.C., Q.M., D.R., and K.L. performed research; K.L. contributed unpublished reagents/analytic tools; H.L., D.W., and X.W. analyzed data; H.L., R.L.B., and J.L. wrote the paper.

This work was supported by NINDS Grant K25NS069805, NIA Grant AG021910, NCRR Grant P41RR14074, and the Howard Hughes Medical Institute. We thank Fenna Krienen, Avram Holmes, and Jorge Sepulcre for assistance with data analysis, Judith Schaechter for discussion of behavioral assessment, Jeremy Schmahmann for discussion of anatomy, and Haderer \& Müller Biomedical Art for illustration.

*J.L. and H.L. contributed equally to this work.

Correspondence should be addressed to either of the following: Dr. Kuncheng Li, Department of Radiology, Xuanwu Hospital of Capital Medical University, Beijing 100053, China, E-mail: likuncheng1955@yaho0.com.cn; or Dr. Randy Buckner, Psychology and Center for Brain Science, Harvard University, Cambridge, MA 02138, E-mail: randy_buckner@harvard.edu.

DOI:10.1523/JNEUROSCI.2364-11.2011

Copyright $\odot 2011$ the authors $\quad 0270-6474 / 11 / 3115065-07 \$ 15.00 / 0$ velopment, advanced aging, and in brain disease (Greicius, 2008; Fornito and Bullmore, 2010; Fox and Greicius, 2010; Vogel et al., 2010; Zhang and Raichle, 2010).

Recent studies further suggest that intrinsic functional connectivity reflects polysynaptic connections. A particularly interesting observation is that intrinsic functional connectivity reveals cerebrocerebellar circuits that are exclusively polysynaptic (Habas et al., 2009; Krienen and Buckner, 2009; O’Reilly et al., 2010; Buckner et al., 2011). Intrinsic fluctuations in the motor cortex, for example, are functionally coupled to their topographically appropriate contralateral regions in the anterior lobe of the cerebellum (Buckner et al., 2011). A direct test of the relation between fcMRI and anatomic connectivity thus would be to examine functional connectivity in cases in which anatomical pathways are selectively disrupted. A recent study showed that severing the corpus callosum reduces functional correlation between homologous regions in the left and right hemispheres (Johnston et al., 2008).

In the present study, we used the well-characterized anatomy of the cerebro-ponto-cerebellar pathway to directly test whether functional connectivity is dependent on a specific polysynaptic pathway (Evarts and Thach, 1969; Kemp and Powell, 1971; Strick, 1985; Schmahmann and Pandya, 1997). Cortical areas project predominantly to the contralateral cerebellum via efferents to the pons and then to the cerebellar cortex. Afferents pass through the deep cerebellar nuclei and then to the thalamus (see Fig. 3A). These connections are organized as closed, parallel circuits: neocortical areas receive input from the same cerebellar 
Table 1. Demography and clinical characteristics of the patients

\begin{tabular}{|c|c|c|c|c|c|c|}
\hline Subject & Gender & Age (years) & Handedness & Lesion side & Lesion description & Symptoms \\
\hline 045 & $\mathrm{~F}$ & 34 & $\mathrm{~L}$ & L (mainly) & L PVTML R PVM & R hemiparesis, dysarthria \\
\hline 024 & M & 48 & $\mathrm{R}$ & L & PVU & R hemiparesis, dysarthria \\
\hline 022 & M & 59 & R & $\mathrm{L}$ & PVML & R hemiparesis, dysarthria \\
\hline 096 & M & 62 & $\mathrm{R}$ & L & PVL & R hemiparesis, dizziness \\
\hline 015 & $\mathrm{~F}$ & 54 & $\mathrm{R}$ & $\mathrm{R}$ & VLU & L hemiparesis \\
\hline 084 & M & 56 & $\mathrm{R}$ & R & PVML & L hemiparesis \\
\hline 021 & M & 57 & $\mathrm{R}$ & $\mathrm{R}$ & PVTML & L hemiparesis \\
\hline 057 & M & 60 & $\mathrm{~L}$ & $\mathrm{R}$ & PVTUM & L hemiparesis, dizziness \\
\hline 072 & M & 61 & $\mathrm{R}$ & R & PVML & L hemiparesis, dysarthria \\
\hline 038 & M & 63 & R & R & PVU & L pure motor hemiparesis \\
\hline 090 & M & 68 & $\mathrm{R}$ & $\mathrm{R}$ & PVL & L pure motor hemiparesis, dizziness \\
\hline
\end{tabular}

F, Female; M, male; L, left; $R$, right.

Table 2. Motor performance measured during five visits for each subject

\begin{tabular}{|c|c|c|c|c|c|c|c|c|c|c|}
\hline Subject ID & FM1 & FM2 & FM3 & FM4 & FM5 & FMC1 & FMC2 & FMC3 & FMC4 & FMC5 \\
\hline 045 & 25.0 & 97.0 & 93.9 & 100.0 & N/A & 0 & 5 & 5 & 6 & $\mathrm{~N} / \mathrm{A}$ \\
\hline 024 & 50.0 & 68.2 & 75.8 & 74.2 & 78.0 & 3 & 3 & 3 & 5 & 6 \\
\hline 022 & 66.7 & 81.8 & 86.4 & 100.0 & 97.0 & 3 & 4.5 & 5 & 6 & 6 \\
\hline 096 & 72.7 & 90.2 & 98.5 & 90.9 & 98.5 & 6 & 6 & 6 & 5 & 6 \\
\hline 015 & 75.0 & 90.9 & 95.5 & 98.5 & 100.0 & 3 & 4 & 5 & 6 & 6 \\
\hline 084 & 44.7 & 14.4 & 40.2 & 43.2 & 57.5 & 1 & 0 & 0 & 3 & 2 \\
\hline 021 & 88.6 & 94.7 & 96.2 & 100.0 & 99.2 & 4 & 4 & 4.5 & 6 & 6 \\
\hline 057 & 42.4 & 59.9 & 84.9 & 98.5 & 100.0 & 0.5 & 1 & 4 & 6 & 6 \\
\hline 072 & 75.0 & 90.9 & 99.2 & 98.5 & 97.0 & 1 & 5 & 6 & 6 & 5 \\
\hline 038 & 62.9 & 83.3 & 96.2 & 100.0 & 100.0 & 2 & 2 & 3.5 & 6 & 6 \\
\hline 090 & 100.0 & 100.0 & 100.0 & 100.0 & 100.0 & 6 & 6 & 6 & 6 & 6 \\
\hline
\end{tabular}

FM, Fugl-Meyer score; FMC, motor coordination score; the numbers indicate visit number (e.g., FM2 is the second visit). Each score represents the average score of two independent raters.

regions to which they project (Middleton and Strick, 2001). A recent anatomical study revealed that the pontocerebellar fibers cross the midline horizontally and then disperse widely in the opposite hemipons before regrouping in the brachium pontis (Schmahmann et al., 2004a). Because of this anatomical arrangement of pontocerebellar fibers, focal lesions to the pons (which arise from infarcts involving small penetrating branches of the basilar artery) disrupt the efferent connections to the contralateral cerebellum but mostly spare ipsilateral connections (Schmahmann et al., 2004a,b). Lesions of the basilar pons thus create a rare opportunity to study the effects on functional coupling after disruption of a specific anatomic pathway.

We investigated the resting-state functional correlation between the cerebral cortex and the cerebellum in first-episode stroke patients (Table 1). The subjects were scanned five times during a period of 6 months. Seven subjects had right pontine infarcts, and four had left infarcts. All subjects had unilateral motor deficits at the acute stage. As a control group, 11 healthy matched subjects were scanned three times within 1 month.

\section{Materials and Methods}

Participants. Eleven first-episode stroke patients (34-68 years old; mean age, 56.6 years; nine male; nine right-handed) with isolated pontine infarcts were recruited from the neurological service at Xuanwu Hospital for a longitudinal investigation. Seven subjects had right pontine infarcts, and four had left infarcts. All 11 patients presented with lateralized hemiparesis. One patient had pure motor hemiparesis (subject 38). Four patients had dysarthria and hemiparesis (subjects 22, 24, 45, and 72). One patient had dizziness and pure motor hemiparesis (subject 90). Two patients had dizziness and hemiparesis (subjects 96 and 57). Three patients only had lateral hemiparesis (subjects 15, 21, and 84). The demographics and clinical characteristics of the patients are summarized in Table 1 . The criteria for enrollment were as follows: fully obtained admission history (within $7 \mathrm{~d}$ after onset of symptoms), single infarction confined to the pons identified on MRI, and no other concomitant brain lesion or previous infarcts. The exclusion criteria were as follows: contraindications for MRI, unclear onset of symptoms, lesions outside the pons or extensive infarcts involving the midbrain or the medulla, recurrence infarction or secondary hemorrhage during follow-up, deafness and/or blindness, aphasia, or a visual field deficit. Eleven healthy control subjects (matched to the patients on age, gender, and handedness) were recruited and scanned three times within 1 month (between-scan interval was $15 \mathrm{~d}$ ). Written informed consent was obtained from all participants under the guidelines of Xuanwu Hospital of Capital Medical University.

MRI data acquisition. Patients were scanned five times during a period of 6 months $(7,14,30,90$, and $180 \mathrm{~d}$ after stroke onset). Scanning was performed on a 3 tesla TimTrio system (Siemens) using the 12-channel phased-array head coil supplied by the vendor. Structural images were acquired using a sagittal MP-RAGE three-dimensional T1-weighted sequence (TR, $1600 \mathrm{~ms}$; TE, $2.15 \mathrm{~ms}$; flip angle, $9^{\circ} ; 1.0 \mathrm{~mm}$ isotropic voxels; FOV, $256 \times 256)$. Each MRI session involved two to four runs $(360 \mathrm{~s} \mathrm{each}$ run) of resting-state fMRI. Subjects were instructed to stay awake and keep their eyes open; no other task instruction was provided. Images were acquired using the gradient-echo echo-planar pulse sequence (TR, 3000 $\mathrm{ms}$; TE, $30 \mathrm{~ms}$; flip angle, $90^{\circ}$; $3 \mathrm{~mm}$ isotropic voxels).

Behavioral examinations. During each visit, behavioral assessments were performed by two clinicians independently before and after the MRI examination. The two scores were averaged to provide a best estimate (Table 2). Motor function, balance, sensation, and joint function of the upper limbs were assessed using 33 tasks (Fugl-Meyer et al., 1975; Gladstone et al., 2002). Each task was rated on a scale of 0 to 2 ( 0 indicates the subject was unable to perform the task, 1 indicates the subject could partially perform the task, and 2 indicates the subject was able to perform the task). The sum of the 33 scores was then normalized to a score between 0 and 100, where 100 represents good performance across all 33 tasks (see FM in Table 2). Finally, the three tasks associated with motor coordination were used to calculate a motor coordination subscore (ranging from 0 to 6 , where 6 represents good performance; see FMC in Table 2). 

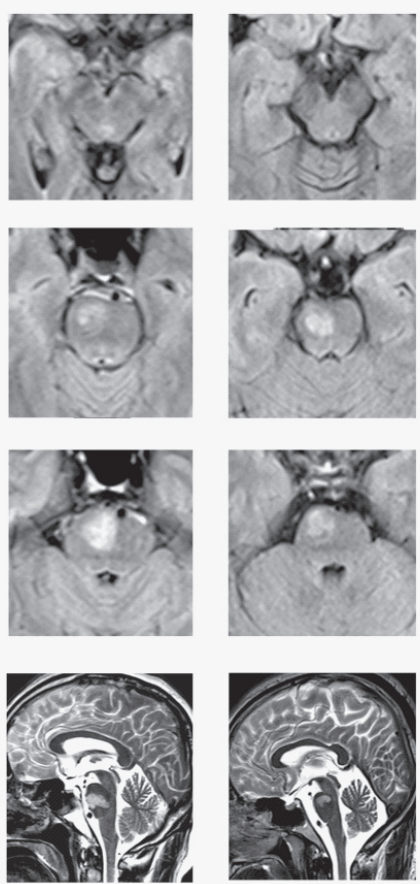

022

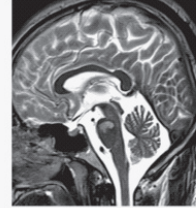

024
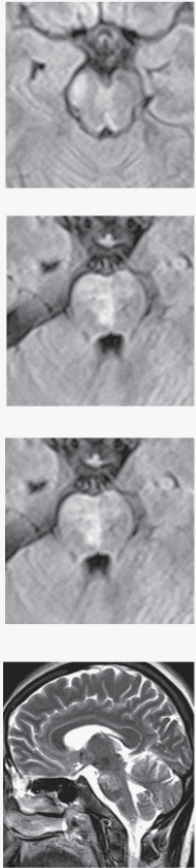

045
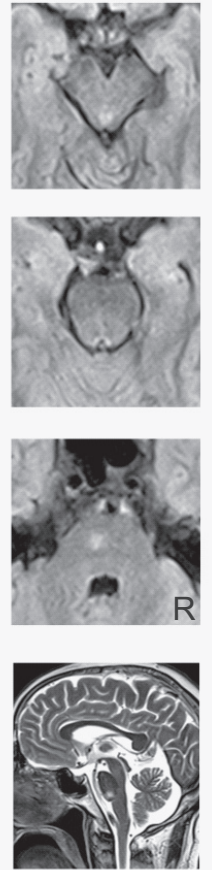

096
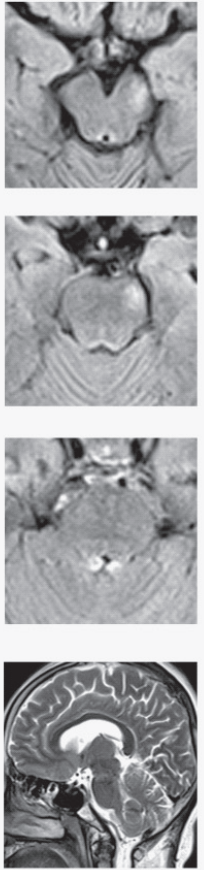

015
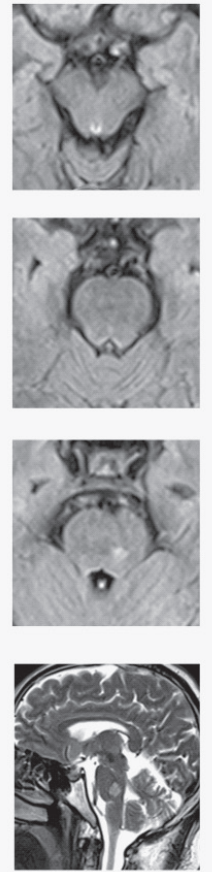

021
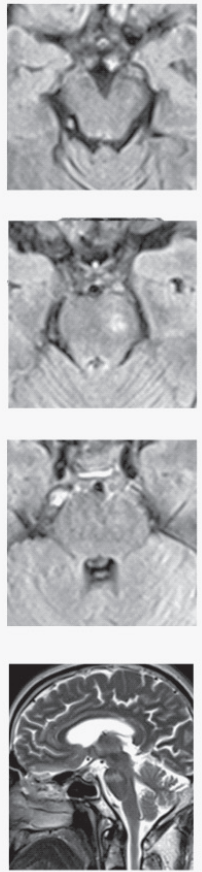

038
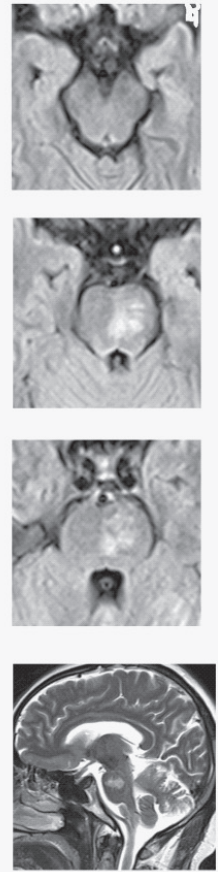

057
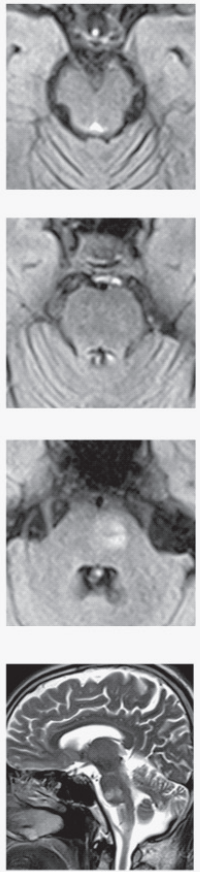

072
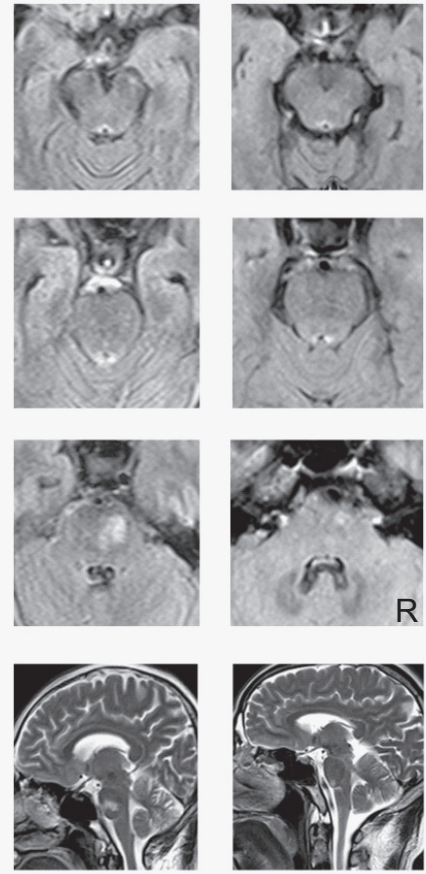

084

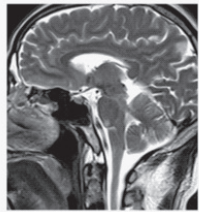

090

Figure 1. Lesion locations are illustrated in three axial planes (from top to bottom) and a sagittal plane for each individual participant. Right is displayed on the right (neurological convention).

National Institutes of Health Stroke Scale (Brott et al., 1989), the modified Rankin scale (van Swieten et al., 1988), and the Barthel index (Granger et al., 1979) were also measured to evaluate the patient's neurological deficit, motor deficit, and life independence.

Description of infarcts. The locations of the infarcts are illustrated in Figure 1 (the overlap of the infarcts across subjects can be seen in Fig. $3 C)$. Neurological convention was used to display the brain images throughout this study. The infarcts were classified into three types based on the MRI examination. (1) Unilateral paramedian ventral pontine infarction was observed in seven patients. Among them, three patients (subjects 22, 72, and 84) had an infarct in the middle and lower (PVML), two patients (subjects 24 and 38) in the upper (PVU), and two patients (subjects 90 and 96) in the lower (PVL) level of the pons. (2) Unilateral ventrolateral upper pontine (VLU) infarction was observed in one patient (subject 15). (3) Unilateral paramedian ventrotegmental pontine (PVT) infarction was seen in three patients. The infarction extended from the basal surface of the pons to the ventral portion of the fourth ventricle. PVT middle and lower (PVTML) and PVT upper and middle (PVTUM) levels of the pons were involved in subjects 21 and 57, respectively. The remaining patient (subject 45) had a larger infarction mainly located in the left PVTML, and part of the right paramedian ventral middle (PVM) was 

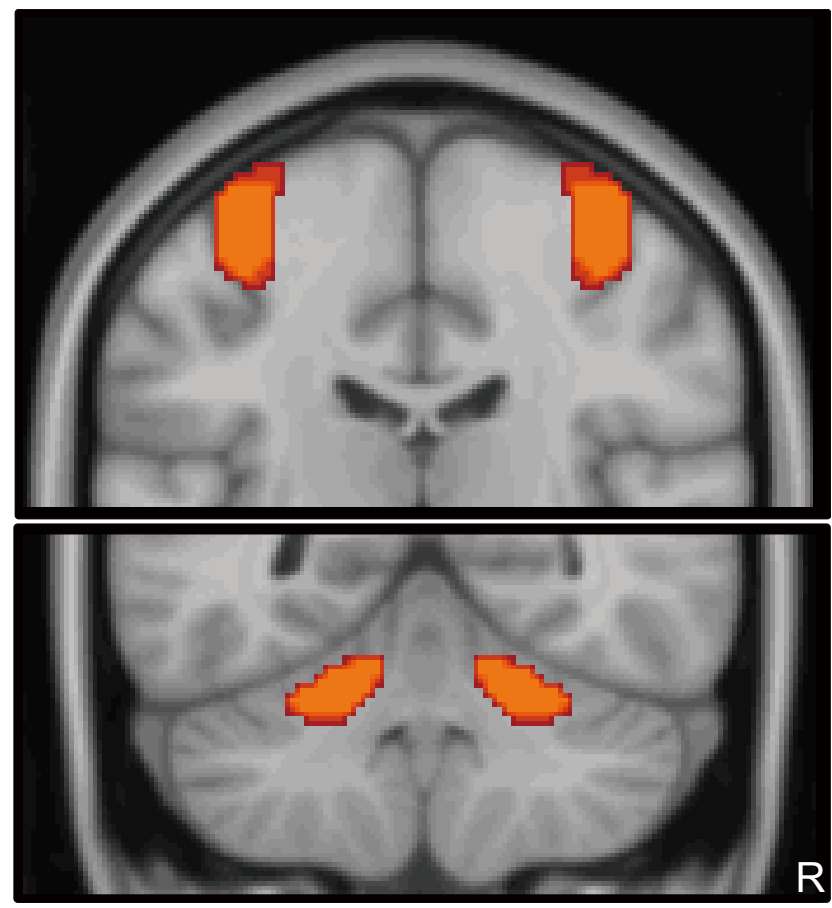

Figure 2. Cerebral and cerebellar motor regions defined by an actual hand movement task. These a priori regions were used for all quantitative analyses. $R$, Right.

involved. Thus, in this patient, the lesion extended beyond that initially estimated at enrollment.

Volumes of the pontine infarctions were measured in diffusionweighted images acquired during the first visit. The size of the infarctions ranged from 2.44 to $29.70 \mathrm{ml}$ (mean, $13.70 \pm 9.86 \mathrm{ml}$ ). The infarctions involving PVL (subjects 90 and 96) were small (2.44 and $4.85 \mathrm{ml}$, respectively). Subject 45 had the largest infarction $(29.70 \mathrm{ml})$ involving left PVTML and a small part of right PVM.

MRI data analysis. Resting-state fMRI data were pre-processed using previously described procedures (Vincent et al., 2006; Van Dijk et al., 2010), which were adapted from Biswal et al. (1995) and Fox et al. (2005). The following steps were performed: (1) slice timing correction (SPM2; Wellcome Department of Cognitive Neurology, London, UK), (2) rigid body correction for head motion, (3) normalization for global mean signal intensity across runs, and (4) transformation of the data into a standard atlas space. The strength of functional correlation between the motor cortex and the contralateral cerebellum was quantified for each patient using hand motor seed regions defined from an independent study of actual hand movements (Buckner et al., 2011; Yeo et al., 2011).

Specifically, 26 young healthy subjects performed a blocked-task paradigm involving right hand movements in MRI scanner. The task consisted of active movement blocks (40 s) interspersed with blocks of passive fixation (18 s). Thirty-six task blocks were acquired for each participant across six runs, 18 blocks of which included right hand movements. A $2 \mathrm{~s}$ visual cue presented before the movement block informed the subjects to initiate the movement. Then, subjects sequentially raised their thumb, index finger, and little finger for the duration of the block. At the end of the block, the subjects were cued to passively fixate. In the present study, brain regions demonstrating significant activation in left primary motor cortex and right cerebellum were identified. The mirrored regions in the opposite hemisphere were then taken for the right cerebrum and left cerebellum (Fig. 2).

Correlation maps were computed for the two cerebral seed regions for each participant. The group-averaged, Fisher's $r$-to- $z$ transformed correlation map was then generated for each seed region. To test for crossed laterality, direct comparisons of the left and right cerebral seed regions were computed by means of arithmetic subtraction of the $z$-score correlation maps. In this manner, lateralization of the cerebrocerebellar con- nectivity could be easily appreciated (Krienen and Buckner, 2009, their Fig. 1).

Correlations among these four seed regions were calculated (left and right for both the cerebrum and the cerebellum). The time course in each region was derived by taking the mean signal within the region over time after preprocessing. Correlations between left cerebral cortex and right cerebellum (LR), right cerebral cortex and left cerebellum (RL), and left and right cerebral motor seed regions (MM) were then calculated for each participant. The $r$-to- $z$ transform was applied to the correlation values before random-effects statistical analysis.

\section{Results}

Functional connectivity reveals contralateral cerebrocerebellar circuits

Cerebrocerebellar connectivity was first explored in healthy control subjects. Hand motor seed regions in both hemispheres were defined from an independent fMRI study (see Materials and Methods and Fig. 2). Subtraction between the two connectivity maps revealed the expected contralateral cerebrocerebellar connectivity (Fig. $3 B$ ). Seed regions in the primary hand motor cortex correlated with the contralateral anterior lobe in lobules IV-VI in the cerebellum and with VIIIB in the posterior lobe, consistent with previous findings (Krienen and Buckner, 2009).

\section{Lesions to the basilar pons disrupt functional connectivity}

Intrinsic functional connectivity was examined in patients with lateralized pontine lesions (lesion locations illustrated in Fig. 3C; see also Fig. 1). Based on the anatomical characteristics of the cerebro-ponto-cerebellar circuit, we hypothesized that lateralized, focal lesions to the basilar pons would disrupt the functional connectivity between the ipsilesional motor cortex and the contralesional cerebellum but spare the connectivity of the contralateral pathway. For initial analyses, the multiple MRI sessions were averaged within subject to boost the stability of the estimate at the expense of capturing variance attributable to recovery process, which is addressed later. Functional connectivity maps were first generated based on seed regions in the left and right motor cortex. Patients with left and right pontine lesions were visualized separately.

The correlation between left motor cortex and right cerebellum (LR) was reduced by left pontine lesions, whereas correlation between the right motor cortex and left cerebellum (RL) remained strong. This pattern was reversed in the patients with right pontine lesions. The cerebrocerebellar functional connectivity maps from several individual subjects clearly showed this pattern of disruption (Fig. 3D), suggesting that intrinsic functional connectivity in this polysynaptic network is sensitive to the integrity of its specific anatomical pathway. Cerebrocerebellar connectivity maps were then averaged across subjects for the left lesion group, right lesion group, and healthy control group (Fig. $4 A$ ). The average maps indicated that cerebrocerebellar functional connectivity was reduced in the lesioned circuit but remained strong in the unaffected circuit, both for left and right lesion groups.

The cerebrocerebellar connectivity was then quantified for each subject. In control subjects, the cerebrocerebellar connectivity of the two circuits (LR and RL) was approximately symmetric at the group level (Fig. $4 B$, middle plot, $t_{(10)}=-1.48, p=$ $0.17)$. In patients with lateralized pontine lesions, the predicted asymmetric cerebrocerebellar connectivity was observed in all but one of the individual subjects. The cerebrocerebellar connectivity pattern was reversed between the left and the right lesion subjects (Fig. $4 B$ ). 

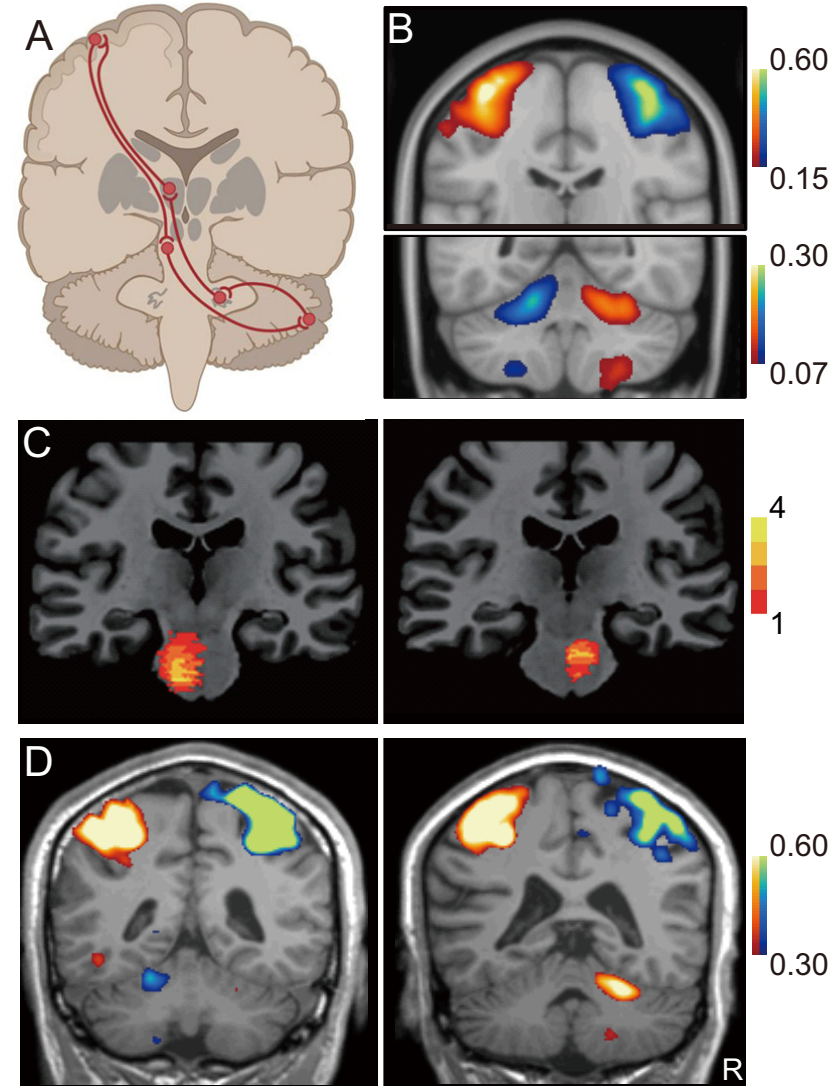

0.60

Figure 3. Pontine lesions disrupt contralateral cerebrocerebellar circuits. A, A schematic illustration of the relevant components of the cerebrocerebellar circuit shows a (simplified) depiction of the polysynaptic efferent pathway between the cerebral cortex and the pons, which then projects preferentially to the contralateral cerebellum. The afferent pathway projects first to the deep cerebellar nuclei and then to the thalamus and the cerebrum. Focal lateralized lesions to the pons preferentially disrupt the efferent pathway from the cerebrum to the cerebellum. $\boldsymbol{B}$, Intrinsic functional connectivity reveals the contralateral cerebrocerebellar connectivity in 11 healthy control subjects. Correlation maps based on left and right motor seed regions (top section, $y=-20, \mathrm{MNI}$ coordinate) were contrasted to reveal contralateral cerebellar regions in the primary and secondary somatomotor representations within the cerebellum (bottom section, $y=-52$ ). C, Distribution of stroke lesions in left lesion (left) and right lesion participants (right). Color scale indicates number of subjects with a lesioned voxel. $\boldsymbol{D}$, Cerebrocerebellar functional connectivity from two individual participants with left-lateralized (left) and right-lateralized (right) lesions. Functional connectivity strength was reduced in the lesioned circuit but remained strong in the unaffected circuit (D). R, Right.

To statistically test the effect, we combined all of the patients into a single group that had two measured values: one for the lesioned circuit ("lesioned") and one for the unaffected circuit ("unaffected"). Note that, for some of these patients, the lesioned circuit involved the right cerebellum and in others the left cerebellum. To accommodate this diversity, we yoked the 11 healthy control subjects to the patients, meaning that for each patient, control measures for the lesioned and unaffected circuits were obtained for the same hemispheres as in the healthy control subjects. This allowed us to test the interaction between circuit (lesioned vs unaffected) and group (patient vs healthy control) while holding lesion laterality constant between groups.

The interaction effect was significant $\left(F_{(1,20)}=9.2, p<0.01\right)$. Decomposing the effect, there was a main effect of circuit (lesioned vs unaffected) for the patients $\left(t_{(10)}=-3.3, p<0.01\right)$ but not for the controls $\left(t_{(10)}=0.58, p=0.57\right)$. Moreover, connectivity of the lesioned circuit in the patients was significantly re-
A

Right
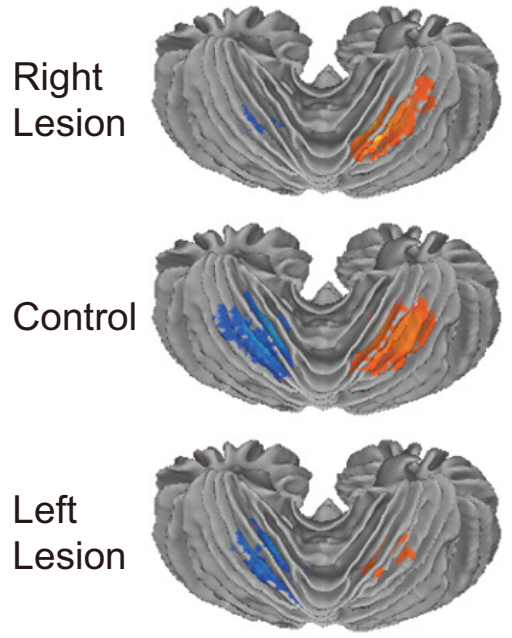

$\mathrm{B}$

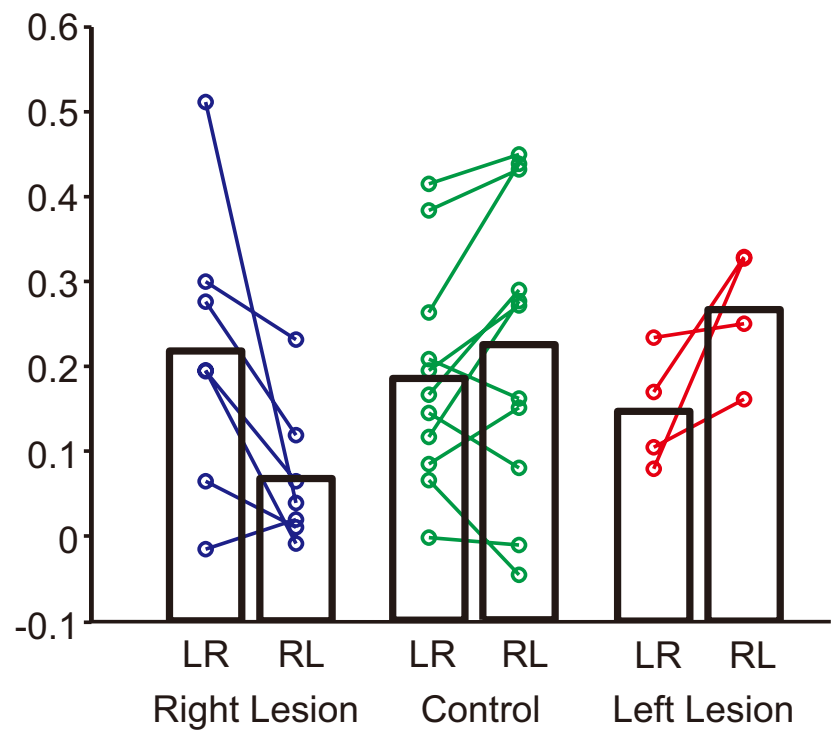

Figure 4. Quantitative analysis of functional connectivity disruption in patients. $A$, Cerebellar functional correlation to hand motor regions in the right lesion group, the healthy control group, and the left lesion group show reduced connectivity in the lesioned circuit. Functional connectivity in the unaffected circuit was at a level similar to that of healthy subjects. The functional connectivity maps are projected to a surface view of the cerebellum exposing the anterior lobe (in which the primary somatomotor representation is visible). $\boldsymbol{B}$, Functional connectivity strength is illustrated for patients and controls. Each line represents an individual participant with the circles reflecting their functional connectivity strength between the left cerebral cortex and right cerebellum (LR) or right cerebral cortex and left cerebellum (RL). Bars are the mean correlation values within each subject group.

duced compared with the matched circuit in the healthy controls (unpaired $t_{(20)}=2.4, p<0.05$ ), whereas the unaffected circuit in the patients showed no difference to the matched circuit in healthy controls (unpaired $t_{(20)}=-0.34, p=0.73$ ).

Patients were scanned five times after their stroke during a period of 6 months. For each of the five separate sessions, we compared the cerebrocerebellar connectivity of the unaffected circuit with the lesioned circuit (Fig. 5). We found that the connectivity strength in the unaffected circuit was stronger than the lesioned circuit across all time points. The effect was significant or showed a trend in four of the five time points $(p=0.04,0.08$, $0.01,0.01,0.21$ for the five time points, respectively). This analysis illustrates that the observations do not depend on averaging the multiple MRI sessions. 


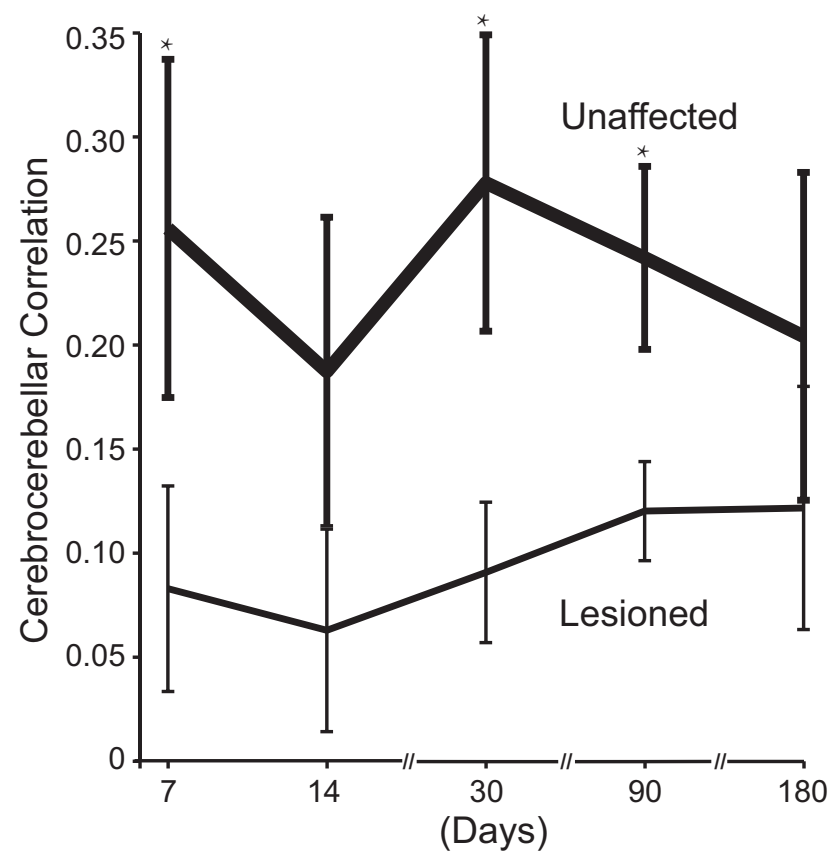

Figure 5. Cerebrocerebellar connectivity of the unaffected circuit (blue) is compared with the lesioned circuit (red) for each scanning session. The scanning sessions are labeled by the time after the stroke ( $x$-axis label). Functional connectivity strength was averaged across all 11 subjects. Connectivity strength in the unaffected circuit was stronger than the lesioned circuit at all time points ( ${ }^{*} p<0.05$, see statistical analysis in Results). Vertical bars represent SE.

As a final analysis, functional connectivity between the left and right cerebral motor seed regions (MM) was also assessed. We found that the M1-to-M1 correlation showed no difference between the patient and the control group $\left(t_{(20)}=0.13, p=\right.$ 0.90 ), indicating that lateralized pontine lesion did not affect the hemispheric correlation in primary motor cortex, although the cerebrocerebellar connectivity was disrupted.

\section{Lesions to the basilar pons may disrupt non-motor pathways} Our initial analyses focused on cerebrocerebellar motor circuits. However, the cerebral cortex forms circuits with the cerebellum that include non-motor pathways. Recent fcMRI studies have revealed specific cerebrocerebellar circuits that involve prefrontal regions and the contralateral cerebellum near Crus I and Crus II (Habas et al., 2009; Krienen and Buckner, 2009; O'Reilly et al., 2010; Buckner et al., 2011). These circuits in the human are predicted by transneuronal tracing techniques in the monkey (Middleton and Strick, 2001) and involve paramedian and peripeduncular nuclei that are near the midline of the pons (Schmahmann and Pandya, 1997). The pontine lesions would thus be expected to also affect these circuits.

For this reason, we conducted a post hoc test to determine whether lateralized pontine lesions also disrupt the functional connectivity between dorsolateral prefrontal cortex (DLPFC) and the cerebellum. Bilateral 8-mm-radius, spherical DLPFC seed regions (MNI coordinates: $\pm 42,16,36$; coordinates reflect the centers of the regions) were defined based on a previous study (Krienen and Buckner, 2009). Functional connectivity maps of these two seed regions were then subtracted.

Similar to the motor pathway, we found that correlation between the left DLPFC and the right cerebellum was reduced by left pontine infarction, whereas correlation between the right DLPFC and left cerebellum remained strong. This pattern was reversed in the patients with right pontine lesions (Fig. 6). These

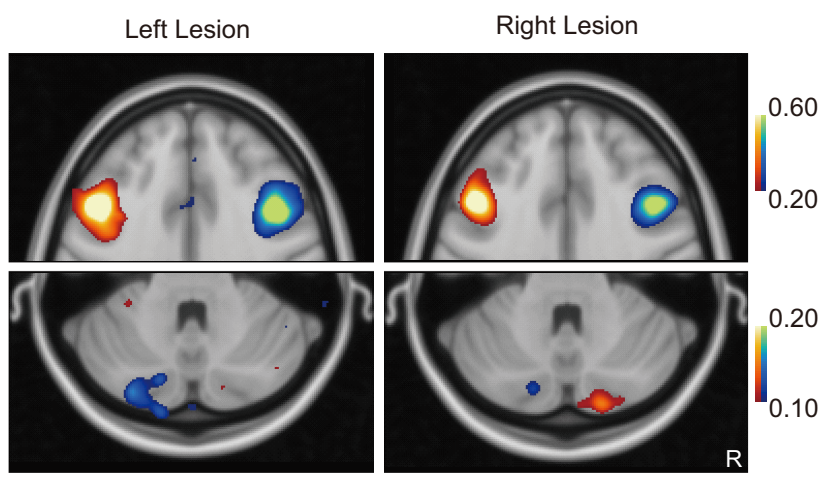

Figure 6. Pontine lesions also disrupt prefrontal-cerebellar circuits. Cerebellar functional correlations contrasting the right and left DLPFC regions in the left lesion group (left) and the right lesion group (right) both show reduced connectivity in the lesioned circuit. Images are transverse sections selected to contain the prefrontal seed regions (top sections, $z=36$, MNI coordinate), as well as the sections capturing the cerebellar correlations (bottom sections, $z=$ -32). Note that the cerebellar correlations fall near Crus I/Crus II, the expected location of prefrontal cerebrocerebellar circuits. The locations of cerebellar regions coupled to prefrontal cortex are anatomically distinct from the somatomotor regions displayed in Figure 3. R, Right.

results suggest that functional connectivity between the DLPFC and the cerebellum also depends on the integrity of the corticoponto-cerebellar circuit.

\section{Discussion}

Analysis of intrinsic functional connectivity has emerged as a powerful tool to study the organization of human brain systems. An open question is whether functional connectivity measured by resting-state fMRI reflects the characteristics of brain circuits defined by anatomical connections. Here we explored this question by examining functional connectivity in patients with focal pontine lesions that disrupt cerebrocerebellar circuitry. We found that functional connectivity between the cerebral cortex and the contralateral cerebellum was disrupted after lateralized, focal infarcts of the basilar pons. Disruption of functional connectivity was observed for the affected side but not the unaffected side. Furthermore, functional coupling between M1 regions between the two hemispheres was at the same level of control subjects.

Although factors beyond anatomical connectivity contribute to fMRI measures of functional correlation (Fox and Raichle, 2007; Moeller et al., 2009; Buckner, 2010), these results provide direct evidence that functional connectivity within a specific polysynaptic circuit is dependent on the integrity of the anatomic pathway. Functional connectivity may be dependent on anatomic connectivity, whereas other factors such as experiencedependent change in synaptic efficacy and momentary responses to task demands further modify functional coupling strength.

The results also raise the possibility of using functional connectivity as a clinical measure. Motor impairment including weakness was observed in all patients during acute stage. Motor recovery was observed in most patients over the 6 month longitudinal window. A future direction for this work will be to explore whether functional connectivity associates with behavioral impairment and can predict recovery. In pursuing this work, it will be important to examine functional connectivity measures of the multiple pathways that are disrupted by focal lesions to the basilar pons. Pontine lesions disrupt the cerebrocerebellar pathway studied here as well as cerebrospinal fibers (Schmahmann et al., 2004a,b). The weakness observed in our patients is likely 
caused (in whole or in part) by disruption of the cerebrospinal pathway.

\section{References}

Biswal B, Yetkin FZ, Haughton VM, Hyde JS (1995) Functional connectivity in the motor cortex of resting human brain using echo-planar MRI. Magn Reson Med 34:537-541.

Brott T, Adams HP Jr, Olinger CP, Marler JR, Barsan WG, Biller J, Spilker J, Holleran R, Eberle R, Hertzberg V (1989) Measurements of acute cerebral infarction: a clinical examination scale. Stroke 20:864-870.

Buckner RL (2010) Human functional connectivity: new tools, unresolved questions. Proc Natl Acad Sci U S A 107:10769-10770.

Buckner RL, Krienen FM, Castellanos A, Diaz JC, Yeo BT (2011) The organization of the human cerebellum estimated by intrinsic functional connectivity. J Neurophysiol. Advance online publication. Retrieved September 12, 2011. doi:10.1152/jn.00339.2011.

Cole DM, Smith SM, Beckmann CF (2010) Advances and pitfalls in the analysis and interpretation of resting-state fMRI data. Front Syst Neurosci $4: 8$.

Evarts EV, Thach WT (1969) Motor mechanisms of the CNS: cerebrocerebellar interrelations. Annu Rev Physiol 31:451-498.

Fornito A, Bullmore ET (2010) What can spontaneous fluctuations of the blood oxygenation-level-dependent signal tell us about psychiatric disorders? Curr Opin Psychiatry 23:239-249.

Fox MD, Greicius M (2010) Clinical applications of resting state functional connectivity. Front Syst Neurosci 4:19.

Fox MD, Raichle ME (2007) Spontaneous fluctuations in brain activity observed with functional magnetic resonance imaging. Nat Rev Neurosci 8:700-711.

Fox MD, Snyder AZ, Vincent JL, Corbetta M, Van Essen DC, Raichle ME (2005) The human brain is intrinsically organized into dynamic, anticorrelated functional networks. Proc Natl Acad Sci U S A 102:9673-9678.

Fugl-Meyer AR, Jääskö L, Leyman I, Olsson S, Steglind S (1975) The poststroke hemiplegic patient. 1. A method for evaluation of physical performance. Scand J Rehabil Med 7:13-31.

Gladstone DJ, Danells CJ, Black SE (2002) The Fugl-Meyer assessment of motor recovery after stroke: a critical review of its measurement properties. Neurorehabil Neural Repair 16:232-240.

Granger CV, Dewis LS, Peters NC, Sherwood CC, Barrett JE (1979) Stroke rehabilitation: analysis of repeated Barthel Index measures. Arch Phys Med Rehabil 60:14-17.

Greicius M (2008) Resting-state functional connectivity in neuropsychiatric disorders. Curr Opin Neurol 21:424-430.

Greicius MD, Supekar K, Menon V, Dougherty RF (2009) Resting-state functional connectivity reflects structural connectivity in the default mode network. Cereb Cortex 19:72-78.

Habas C, Kamdar N, Nguyen D, Prater K, Beckmann CF, Menon V, Greicius MD (2009) Distinct cerebellar contributions to intrinsic connectivity networks. J Neurosci 29:8586-8594.

Honey CJ, Sporns O, Cammoun L, Gigandet X, Thiran JP, Meuli R, Hagmann P (2009) Predicting human resting-state functional connectivity from structural connectivity. Proc Natl Acad Sci U S A 106:2035-2040.
Johnston JM, Vaishnavi SN, Smyth MD, Zhang D, He BJ, Zempel JM, Shimony JS, Snyder AZ, Raichle ME (2008) Loss of resting interhemispheric functional connectivity after complete section of the corpus callosum. J Neurosci 28:6453-6458.

Kemp JM, Powell TP (1971) The connexions of the striatum and globus pallidus: synthesis and speculation. Philos Trans R Soc Lond B Biol Sci 262:441-457.

Krienen FM, Buckner RL (2009) Segregated fronto-cerebellar circuits revealed by intrinsic functional connectivity. Cereb Cortex 19:2485-2497.

Margulies DS, Vincent JL, Kelly C, Lohmann G, Uddin LQ, Biswal BB, Villringer A, Castellanos FX, Milham MP, Petrides M (2009) Precuneus shares intrinsic functional architecture in humans and monkeys. Proc Natl Acad Sci U S A 106:20069-20074.

Middleton FA, Strick PL (2001) Cerebellar projections to the prefrontal cortex of the primate. J Neurosci 21:700-712.

Moeller S, Nallasamy N, Tsao DY, Freiwald WA (2009) Functional connectivity of the macaque brain across stimulus and arousal states. J Neurosci 29:5897-5909.

O'Reilly JX, Beckmann CF, Tomassini V, Ramnani N, Johansen-Berg H (2010) Distinct and overlapping functional zones in the cerebellum defined by resting state functional connectivity. Cereb Cortex 20:953-965.

Schmahmann JD, Pandya DN (1997) Anatomic organization of the basilar pontine projections from prefrontal cortices in rhesus monkey. J Neurosci 17:438-458.

Schmahmann JD, Rosene DL, Pandya DN (2004a) Ataxia after pontine stroke: insights from pontocerebellar fibers in monkey. Ann Neurol 55:585-589.

Schmahmann JD, Ko R, MacMore J (2004b) The human basis pontis: motor syndromes and topographic organization. Brain 127:1269-1291.

Strick PL (1985) How do the basal ganglia and cerebellum gain access to the cortical motor areas? Behav Brain Res 18:107-123.

Van Dijk KR, Hedden T, Venkataraman A, Evans KC, Lazar SW, Buckner RL (2010) Intrinsic functional connectivity as a tool for human connectomics: theory, properties, and optimization. J Neurophysiol 103:297-321.

van Swieten JC, Koudstaal PJ, Visser MC, Schouten HJ, van Gijn J (1988) Interobserver agreement for the assessment of handicap in stroke patients. Stroke 19:604-607.

Vincent JL, Snyder AZ, Fox MD, Shannon BJ, Andrews JR, Raichle ME, Buckner RL (2006) Coherent spontaneous activity identifies a hippocampalparietal memory network. J Neurophysiol 96:3517-3531.

Vincent JL, Patel GH, Fox MD, Snyder AZ, Baker JT, Van Essen DC, Zempel JM, Snyder LH, Corbetta M, Raichle ME (2007) Intrinsic functional architecture in the anaesthetized monkey brain. Nature 447:83-86.

Vogel AC, Power JD, Petersen SE, Schlaggar BL (2010) Development of the brain's functional network architecture. Neuropsychol Rev 20:362-375.

Yeo BTT, Krienen FM, Sepulcre J, Sabuncu MR, Lashkari D, Hollinshead M, Roffman JL, Smoller JW, Zöllei L, Polimeni JR, Fischl B, Liu H, Buckner RL (2011) The organization of the human cerebral cortex estimated by intrinsic functional connectivity. J Neurophysiol 106:1125-1165.

Zhang D, Raichle ME (2010) Disease and the brain's dark energy. Nat Rev Neurol 6:15-28. 